\title{
Editorial on the Significance of Endovascular Treatment in Coronary, Aorta, Pulmonary Vein Repairs
}

\section{Vincent John*}

Department of Gynaecology and Health Sciences, Faculty of Medicine, Universidade de Sao Paulo, Argentina

*Corresponding author: Vincent John, Department of Gynaecology and Health Sciences, Faculty of Medicine, Universidade de Sao Paulo, Argentina, Tel +5511 3091-3116; E-mail: vincentjohnvj@rediffmail.com

Received date: June 28 2017, Accepted date: July 03 2017, Published date: July 052017

Copyright: 2017 (c) John V. This is an open-access article distributed under the terms of the Creative Commons Attribution License, which permits unrestricted use, distribution, and reproduction in any medium, provided the original author and source are credited.

\section{Editorial}

A 55 year female patient with history of hypertension, dyslipidemia, diabetes mellitus and past myocardial dead tissue (MI) displayed to the crisis office with intense coronary disorder. His past coronary occasion was a substandard MI which was treated with thrombolysis six years back inside 7 hours of onset. Catheterization around then uncovered abnormal right coronary vein beginning from the left valsalva sinus with direct proximal deterrent and the patient gotten restorative treatment. He had as of late played out a clinical assessment with an ordinary treadmill test and typical echocardiogram in another hospital. He introduced to the crisis bureau of a non every minute of every day essential PCI medicinal focus with intense onset (2 hours) of serious chest torment and diaphoresis. On confirmation he was on edge, his circulatory strain was $80 / 50 \mathrm{mmHg}$ and he had typical heart and lung auscultation. His first electrocardiogram demonstrated an ectopic atrial musicality, second rate inert zone and ST rise in precordial leads V1-V3 (there were no past electrocardiogram tracings accessible for correlation). He was treated with IV hydration, acetylsalicylic corrosive $275 \mathrm{mg}$, clopidogrel $450 \mathrm{mg}$ and thrombolysed with Tenecteplase 25 $\mathrm{mg}$ with change of fringe perfusion furthermore, pulse.

Coronary angiography showed a left prevailing framework with obstructive injuries in the second harsh peripheral, ramus intermedius and the proximal left front diving (LAD) supply routes, and in addition the nondominant right coronary. Finish impediment of the infra-renal aorta with patent two-sided renal courses and prevalent mesenteric corridor was noted. Fruitful stenting of LAD, harsh peripheral and ramus intermedius supply routes was performed after 72 hours, the patient griped of intense chest torment a rehash coronary angiogram demonstrated intense stent thrombosis in ramus intermedius requiring rehash angioplasty and stent arrangement. The healing facility course was additionally entangled by intermittent fevers and the patient was found to have declining rhabdomyolysis of the lower furthest points with rising creatinine kinase levels. Persistent proceeded to have fevers notwithstanding anti-infection agents and created compounding left lower limit swelling and vanishing of Doppler signs of the left foot.

In more established cases it is accounted for that a comparative instance of patient with CAD with Leriche disorder, who was moderately stable permitting tending to the aortic impediment as an initial step taken after by complex PCI of the RCA injury. Not at all like our patient, the aortic injury in that patient could be crossed with ordinary approach utilizing bifemoral also, left brachial approach and two self-extending stents were conveyed at the aorto-iliac bifurcation. An investigation by Kim et al examined 49 patients with a CTO of the aorta treated through endovascular approach. They announced procedural accomplishment in 40 cases with 7 patients requiring remediation and an general $80 \%$ patency rate at 3 years. None of the patients required real removal yet major intricacies were accounted for in $16 \%$ of the cases, for example, spinal and cerebral areas of localized necrosis, distal embolization, side branch imprisoning, iliac vein break and pseudoaneurysm arrangement among others.

Some case reports balance the traditional electrocardiogram finding of ST height in DII, DIII and AVF for right coronary intense impediment, a large portion of them being consequence of disengaged right ventricular myocardial localized necrosis (RVMI) when precordial ST height can happen. Strangely, Kanovsky, detailed in an overview of 300 patients with electrocardiographic characterized RVMI (V4R ST fragment rise) that the offender vessel was the correct coronary corridor in just $48 \%$ of the cases, the left foremost plunging conduit in $63 \%$ and the circumflex course in $5 \%$ of cases. One ought to likewise take note of that in this examination RV work was not assessed and we could just deduce it to be more awful in patients with RCA impediment as a result of the more noteworthy size of RV branches from this vessel it has chosen a few angles that would propose RCA impediment/RVMI when precordial ST rise are available: diminishing extent of ST portion rise from V1 to V3, arched ST rise, corresponding sinus hub brokenness and V3-V4l ST rise. Likewise, the creators recommend a corresponding bedside assessment with echocardiography in which RV enlargement would add to the electrocardiogram approach. In the announced case, the patient had a gentle cardiogenic stun introduction because of STEMI with fizzled thrombolysis and clear angiographic meaning of RCA as the guilty party vessel, without noteworthy stenosis on the left coronary conduit. Or maybe critical as well, the RCA impediment was proximal to the RV branches and "confined" RVMI in the setting of a past second rate divider myocardial dead tissue was considered the reason for the precordial ST height without ST rise in drives DII, DIII and AVF. Additionally important, sinus hub brokenness was available as ectopic atrial cadence was appeared at introduction and standardized to sinus cadence simply after reperfusion. A portion of the previously mentioned electrocardiographic components were available, for example, raised ST rise and sinus hub brokenness however diminishing ST rise from V1 to V3 were not found for this situation. Lamentably, in light of the fact that our foundations as it were perform right precordial leads on affirmation in sub-par myocardial areas of dead tissue, we do not have information on V3-V4R drives which we accept would be demonstrating ST rise.

This case represents a mind boggling circumstance of a symptomatic extreme CAD with noteworthy LV dysfunction, AIOD, securities from sidelong thoracic divider supply routes and epigastric corridors to bring down appendage courses convoluted by distal basic lower appendage blood vessel impediment that was overseen by percutaneous intercession of the coronary sores, endovascular opening of CTO of aortic impediment utilizing wildcat catheter and 
Citation: John V (2017) Editorial on the Significance of Endovascular Treatment in Coronary, Aorta, Pulmonary Vein Repairs. Med Rep Case Stud 2: e101. doi:10.4172/2572-5130.1000e101

Page 2 of 2

revascularization of lower appendage blood vessel impediments. Secluded aortopulmonary insurances in a solid neonate with no other intrinsic coronary illness or lung sickness are uncommon. Our patient is the principal answered to have a secluded huge aortopulmonary insurance being the sole pneumonic blood supply to a whole a lung portion. Consequently, we didn't continue with winding of the APC. Due to its irregularity, there is still much to find out about the starting point and advancement of these pledges that perhaps grew prenatally. Albeit extraordinary in proximal RCA impediment, the electrocardiographic example appeared for this situation may prompt incorrect guilty party vessel definition and treatment delay in this serious clinical situation. Exhaustive ECG assessment and integral snappy bedside assessment can enhance finding and abbreviate doorto-swell time and result in better patient anticipation. 